\title{
Disclosure do modelo de economia circular aplicado no mercado de energia em um dos maiores grupos empresariais privados do Brasil: um estudo de caso
}

Disclosure of the circular economy model applied to the energy market in one of the largest private business groups in Brazil: a case study

Divulgación del modelo de economía circular aplicado al mercado energético en uno de los mayores grupos empresariales privados de Brasil: un caso de studio

Recebido: 11/11/2021 | Revisado: 16/11/2021 | Aceito: 22/11/2021 | Publicado: 23/11/2021

Laryssa Kelly de Almeida Virginio
ORCID: https://orcid.org/0000-0001-9750-8203
Universidade Federal de Campina Grande, Brasil
E-mail: laryssa_kellypb @ hotmail.com
Isabel Lausanne Fontgalland
ORCID: https://orcid.org/0000-0002-0087-2840
Universidade Federal de Campina Grande, Brasil
E-mail: isabelfontgalland@ @mail.com

\section{Resumo}

A Raízen é uma referência global em bioenergia, protagonista na transição energética e se destaca na busca por redefinir o futuro da energia. Neste artigo, será abordado o modelo de economia circular aplicado à essa empresa, que tem como objetivo uma gestão mais eficiente dos recursos naturais existentes, ou seja, manter produtos, componentes e materiais em seu mais alto nível de utilidade e valor o tempo todo, dentro de um escopo econômico de desenvolvimento sustentável. O estudo em tela visa discutir como a empresa realiza sinergias buscando um modelo de economia circular eficiente, analisar as práticas divulgadas do modelo de economia circular e os potenciais fatores que influenciam estas, identificar oportunidades de práticas, soluções e as barreiras encontradas, a partir de uma compreensão do modelo de economia circular e dos avanços que este revela para o alcance da sustentabilidade. Espera-se contribuir com futuros trabalhos nessa área, ao possibilitar um olhar mais objetivo e real sobre o assunto, dado a sua relevância e no sentido de contribuir para o alcance do desenvolvimento sustentável.

Palavras-chave: Economia circular; Recursos naturais; Sustentabilidade.

\begin{abstract}
Raízen is a global reference in bioenergy, a protagonist in the energy transition and stands out in the search for redefining the future of energy. In this article, the circular economy model applied to this company will be addressed, which aims at a more efficient management of existing natural resources, that is, to maintain products, components and materials at their highest level of utility and value at all times, within an economic scope of sustainable development. The on-screen study aims to discuss how the company realizes synergies by seeking an efficient circular economy model, analyze the practices disclosed in the circular economy model and the potential factors that influence them, identify practical opportunities, solutions and the barriers encountered, based on an understanding of the circular economy model and the advances it reveals towards achieving sustainability. It is expected to contribute with future works in this area, by enabling a more objective and real look at the subject, given its relevance and in the sense of contributing to the achievement of sustainable development.
\end{abstract}

Keywords: Circular economy; Natural resources; Sustainability.

\section{Resumen}

Raízen es un referente mundial en bioenergía, protagonista en la transición de la energía y se destaca en la búsqueda de redefinir el futuro de la energía. En este artículo se abordará el modelo de economía circular aplicado a esta empresa, que tiene como objetivo una gestión más eficiente de los recursos naturales existentes, es decir, mantener los productos, componentes y materiales en su máximo nivel de utilidad y valor en todo momento, dentro de un ámbito económico de desarrollo sostenible. El estudio en pantalla tiene como objetivo discutir cómo la empresa logra sinergias buscando un modelo de economía circular eficiente, analizar las prácticas en la identificación del modelo de economía circular y los factores potenciales que influyen en ellas, oportunidades prácticas, soluciones y las barreras encontradas, en base a una comprensión del modelo de economía circular y los avances que revela para lograr la sostenibilidad. Se espera que contribuya con los trabajos futuros en esta área, al posibilitar una mirada más objetiva y real al tema, dada su relevancia y en el sentido de contribuir al logro del desarrollo sostenible.

Palabras clave: Economía circular; Recursos naturales; Sustentabilidad. 


\section{Introdução}

O crescimento populacional tem demonstrado aumento do consumo em massa e produção em grande escala, influenciando o bem-estar de todos com esgotamento dos recursos não renováveis, poluição de ar, água e solo. As mudanças climáticas e o aquecimento global são consequências também dessa crescente, e surgem com efeitos iminentemente destrutivos para a humanidade, isto têm alertado governos e empresas a adoção de medidas de mitigação de impactos ambientais junto ao crescimento da economia. Segundo "O Nosso Futuro Comum” conhecido como Relatório Brundtland, o desenvolvimento sustentável, é "aquele que atende às necessidades do presente sem comprometer a possibilidade de as gerações futuras atenderem às suas necessidades" (Comissão Mundial sobre Meio Ambiente e Desenvolvimento, 1988). Por precisão, desde os eventos da época, o tema se apresenta entre os mais discutidos, em torno do ramo de gestão empresarial, compondo assim o pilar da estratégia competitiva das empresas; e tornar sustentável o crescimento das entidades como finalidade. No atual cenário, de não suficiência de qualidade se faz necessário e urgente externar os cuidados, o impacto positivos das suas operações dentro e fora das paredes institucionais.

As ações envolvendo a chamada economia circular (EC), apresentam relevantes inovações, soluções tecnológicas, acompanhadas de campanhas educativas para a atual situação, pois buscam investir no ciclo do produto, mantendo-o o maior tempo possível no processo produtivo, a favor da sustentabilidade. Para isto, a EC visa alterar o sistema produtivo desde da extração, manufatura, consumo e despejo de resíduos, substituindo a economia linear para o sistema circular, por isso deve contar com as inovações, além de possuir boa audiência dos seus princípios no meio corporativo e governamental (RAMOS et al., 2020).

A EC, dentre as suas contribuições para o mercado, tem destacado o propósito de "acabar com ineficiências, ao longo do ciclo de vida do produto, desde a extração das matérias-primas até à sua utilização, pelo consumidor final, através de uma gestão mais eficiente dos recursos naturais, minimizando ou erradicando a criação de resíduos e prolongando, ao máximo, a vida útil e o valor do produto" (Lemos, 2018). Com isso, os produtos desde a fase inicial de fabricação tem-se um ciclo eficiente a cumprir, podendo ser reconduzido e aproveitados na produção, em busca da concepção de não desperdícios ou geração de resíduos.

Segundo Ellen MacArthur Foundation - EMF (2012), a EC baseia-se em três princípios básicos: 1) Preservar e aprimorar o capital natural controlando estoques finitos e equilibrando os fluxos de recursos renováveis; 2) Otimizar o rendimento de recursos fazendo circular produtos, componentes e materiais no mais alto nível de utilidade o tempo todo, tanto no ciclo técnico quanto no biológico; 3) Estimular a efetividade do sistema revelando e excluindo as externalidades negativas desde o princípio. Adotando-os, gestão responsável se faz coeva , eficiente, de relevante preocupação e colaboração com meio ambiente.

Em pesquisa feita pela Confederação Nacional da Indústria (CNI, 2019), foi exposto que 76,5\% das indústrias desenvolvem alguma iniciativa de economia circular, embora a maior parte não saiba que as ações se enquadram nesse conceito. Entre as principais práticas estão a otimização de processos $(56,5 \%)$, o uso de insumos circulares $(37,1 \%)$ e a recuperação de recursos $(24,1 \%)$. A pesquisa também mostra que $88,2 \%$ dos entrevistados avaliaram a economia circular como importante ou muito importante para a indústria brasileira e não se trata apenas da busca por eficiência e economia, mas favorecimento ao meio ambiente (Agência Brasil, 2021).

Diante deste contexto, entende-se que a presente situação não é satisfatória, sobretudo quando se volta a atenção à capacidade das empresas e do meio ambiente de atender às demandas atuais e futuras da sociedade para corresponder às suas exigências. Neste sentido, considerando a relevância do tema e a constante busca mundial pela sustentabilidade, visto que um dos maiores desafios é redução da geração excessiva e disposição final ambientalmente segura dos resíduos, o presente trabalho 
surge para analisar quais as práticas divulgadas do modelo de economia circular de um dos maiores grupos empresariais privados do Brasil, a relevância para o meio ambiente e no mercado.

Em toda cadeia produtiva, o termo sustentabilidade tem assumido papel de destaque, gerando um esforço coletivo para a preservação dos nossos recursos naturais. A EC baseia-se nisso como uma estratégia na busca das melhores condições para o meio ambiente, no tocante que, por hora o que era resíduo, será matéria prima para início de outra produção, de forma circular e proveitosa.

Portanto, é pertinente a preocupação das responsabilidades com os recursos naturais associadas as estratégias adotadas pelas empresas, pois impulsionados pela crescente procura, os produtos diversificam-se rapidamente e segue aumentando consequentemente o montante de insumos extraídos do meio ambiente e dos resíduos produzidos por estes e pela produção. Sendo assim, é fundamental o esclarecimento para os gestores, profissionais da área e todos aqueles que tenham interesse em compreender como funciona a busca pelo modelo de EC eficiente na pratica.

Nesse artigo, será abordado a estratégia da economia circular que tem por objetivo, uma gestão mais eficiente dos recursos naturais existentes, ou seja, manter produtos, componentes e materiais em seu mais alto nível de utilidade e valor o tempo todo, dentro de um escopo econômico de desenvolvimento sustentável. Esse estudo visa discutir como a empresa realiza sinergias buscando um modelo de economia circular eficiente, além disso, analisar as práticas divulgadas do modelo de economia circular e os potenciais fatores que influenciam estas; identificar oportunidades de práticas, soluções e as barreiras encontradas, a partir de uma compreensão do modelo de economia circular e dos avanços que este revela para o alcance da sustentabilidade.

\section{Metodologia}

O referido estudo será baseado, quanto aos métodos, como sendo um estudo de caso, uma vez que consiste na compreensão do fenômeno, utilizando-se comumente de dados qualitativos, coletados a partir de eventos reais. É impreterível cumprir com algumas etapas para realizar pesquisas utilizando o método do estudo de caso, são elas divididas em no mínimo cinco etapas: Planejamento da pesquisa; desenho da pesquisa; preparação e/ou coleta dos dados; análise do caso; e elaboração dos relatórios (Popadiuk, 2015). Para cumprimento dessas etapas, utilizou-se de método documental para o planejamento de pesquisa, (GIL, 2002) tendo em vista que até definir a abordagem e desenhá-la se debruçou-se sobre inúmeras produções acadêmicas. Na coleta de dados, além das anteriormente citadas, utilizando-se também das informações divulgadas no site pela empresa nos últimos anos, em busca de descrever o modelo de economia circular de um dos maiores grupos empresariais privados do Brasil.

$\mathrm{Na}$ análise do caso, tem-se em tela a Raízen Inc. e seus relatórios de holding. Adotou-se a abordagem qualitativa pois busca "[...] descrever a complexidade do problema, compreender e classificar processos dinâmicos vividos por grupos sociais e contribuir no processo de mudança de determinado grupo [...]” (Richardson, 1999). As duas últimas etapas ocorrem paralelamente e não podem ser isoladas. Quanto a natureza, ela se enquadra em uma pesquisa de cunho básico, onde objetiva analisar um caso específico sem a necessidade de aplicação prática.

\section{Resultados e Discussão}

\section{A Raízen}

A Raízen é empresa integrada de energia, originada em solo brasileiro (mas também atua na Argentina), referência global em bioenergia, protagonista na transição energética e se destaca na busca por redefinir o futuro da energia. Está entre os maiores grupos empresariais privados do Brasil, a $2^{\mathrm{a}}$ maior empresa distribuidora de combustíveis, a $4^{\mathrm{a}}$ maior empresa em faturamento no Brasil e possui mais de 40 mil funcionários e 15 mil parceiros de negócios espalhados pelo país segundo dados disponíveis no site Raízen (2021). 
Com suas práticas, impulsiona a sociedade com soluções em energia, apresentando a circularidade em sua produção. Atuando desde a produção e venda de energia renovável e açúcar a partir da cana-de-açúcar, levando essa energia para diversos cantos no mundo, também com uma forte atuação em marketing e serviços. A Raízen, em seu site, descreve seus produtos presentes no açúcar do cafezinho de todas as manhãs, nos produtos de higiene e beleza que trazem o etanol como ingrediente, no combustível de carros e aviões que encurtam distâncias, e na energia elétrica gerada a partir dos subprodutos da cana-deaçúcar. Tudo para fazer a vida e a economia girar.

Além dos aspectos econômico-financeiros, de acordo com os relatórios divulgados, a mesma destaca sempre o comprometimento com a ética, a segurança e a sustentabilidade. Afirma que não há desenvolvimento real se não for sustentável. Externam o conhecimento do quanto comunidades e natureza também dependem da empresa. E por isso, responsabilidade social e ambiental estão na base dos pilares estratégicos, permeando todas as etapas dos processos produtivos e comerciais. Em todas as unidades produtivas da Raízen, são desenvolvidos projetos destinados a mobilizar os funcionários em atividades de cidadania e participação social, priorizando temas que beneficiem a sociedade e estejam alinhados ao negócio.

\section{Economia circular}

A circularidade no processo produtivo faz parte do setor sucroenergético da Raízen que é destaque nesse modelo, apresentando forte característica pela redução da geração de resíduos e a regeneração dos sistemas, utilizando-se da reutilização, recuperação, reciclagem de materiais e energia. Segundo site da Raízen, a pratica dessas características e o cuidado com o meio ambiente começa desde do canavial, que adota subprodutos advindos da própria cana-de açúcar incorporados na terra, fazendo assim uma eficiência no processo, otimização para as novas colheitas, geração de valor, perenidade do negócio e fechando esse ciclo no mesmo local.

Na Figura 1 temos uma explanação de como a empresa realiza sinergias buscando um modelo de Economia Circular eficiente. 
Figura 1: Círculo do produto.

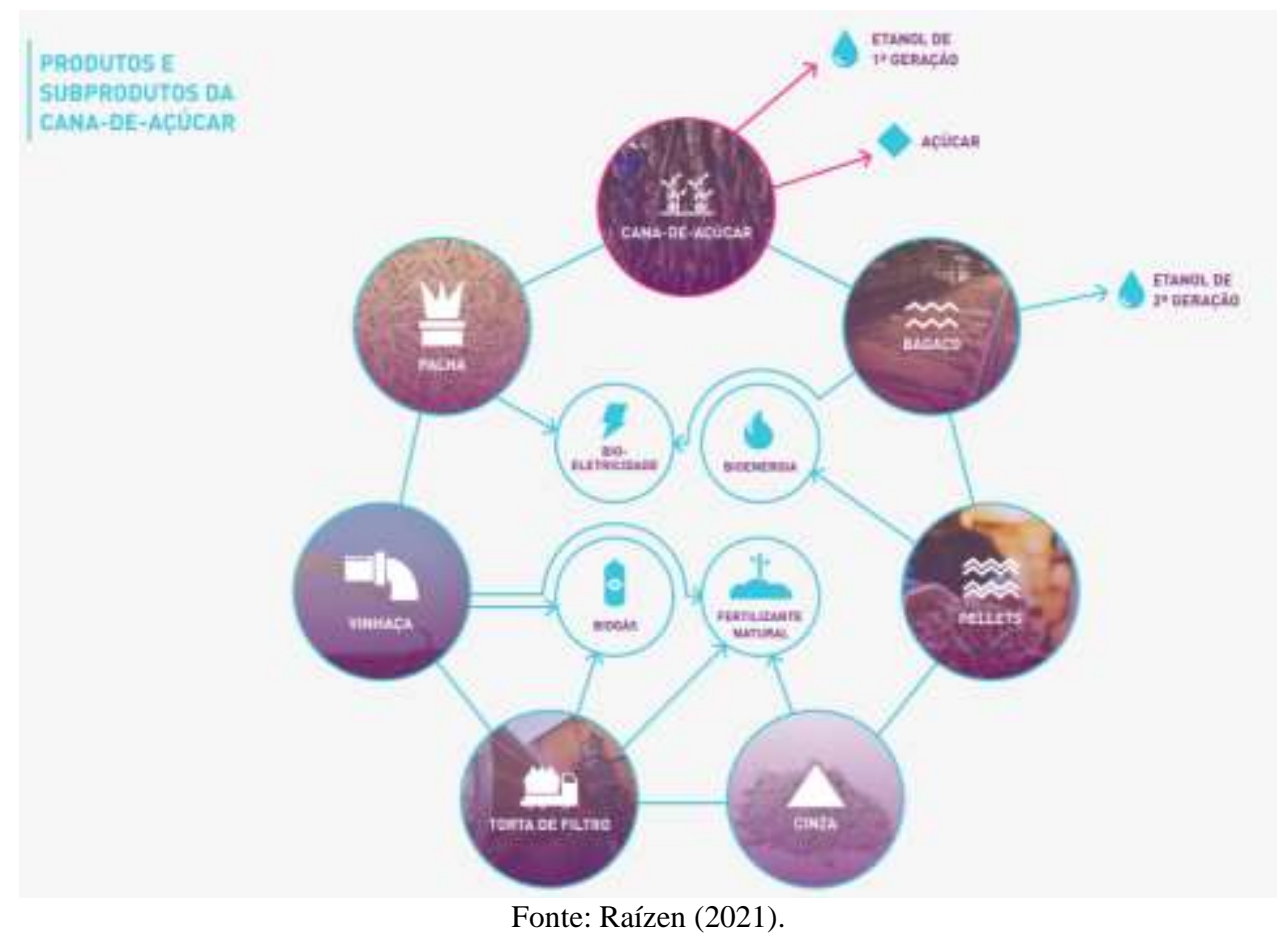

Como pode-se, observar os produtos são elaborados para circular de forma eficiente e serem recolocados na produção, reproduzindo, dessa forma, a concepção de não desperdícios ou resíduos. De início, com a cana-de-açúcar tem-se a produção do etanol de $1^{a}$ geração e o açúcar; e em seguida com o bagaço e a palha gera-se a bioeletricidade. Segundo a Raízen a bioeletricidade garante a autossuficiência energética das usinas, sendo o bagaço usado também para produção de etanol de $2^{\mathrm{a}}$ geração, o E2G, um exemplo inovador de bioeconomia e sustentabilidade aplicada. Pellets são produzidos a partir de biomassa, com uma menor pegada de carbono que seus substitutos convencionais (carvão) além de ser 100\% renovável. Por fim, e início do próximo ciclo a cinza, torta de filtro e vinhaça são usados como fertilizantes naturais, isto é, reduz o uso de fertilizantes sintéticos e voltam para a terra, fechando um círculo nutritivo. Vale ressaltar que a torta de filtro e a vinhaça ainda são usadas também para produzir o biogás, mais uma energia renovável.

De acordo com a Raízen (2021), a empresa utiliza-se da circularidade também na gestão hídrica, buscando reduzir o seu impacto ambiental, nos recursos ligados à gestão da água. Pensando nisso, criou-se o Reduza — programa de gestão hídrica da Raízen, empregando práticas como redução da captação de água de fontes externas, por meio do maior aproveitamento da água presente na própria cana; investimento em tecnologia e boas práticas de reuso dentro das unidades industriais e sempre buscando uma menor pegada hídrica para os produtos. Segundo relatório da Raízen (2021), desde da criação do Reduza, diminuiu-se a dependência e empresa gerou uma economia de água não captada de 27 bilhões de litros, considerando o gasto anterior à criação do programa.

Nas práticas divulgadas, do modelo circular da Raízen, é claro a busca pela sustentabilidade, gerando um empenho coletivo para maior aproveitamento do produto e a preservação dos recursos naturais, de modo a utilizar os materiais de forma repetida em ciclos que mantém seu valor intrínseco, visto que, os maiores desafios na cadeia produtiva é a geração excessiva e disposição final ambientalmente segura dos resíduos sólidos.

São aplicações de estratégias significativas para o meio ambiente a serem encaradas como incentivo para um crescimento com bases solidas, considerando que a sustentabilidade e a valorização dos recursos naturais resultam em melhores perspectivas e condições de saúde aos seres humanos. Assim, há benefícios para o ambiente, para o crescimento econômico e para a população. 


\section{Compromissos ligados à desafios globais}

A EC é consequência da perseverança da empresa no alcance com seus compromissos, no objetivo de intensificar a sua responsabilidade com a agenda de sustentabilidade, e junto com a auditoria independente buscam chegar aos Objetivos de Desenvolvimento Sustentável (ODS) da ONU, para avançar no desenvolvimento e torná-lo realidade. E nesta busca, conforme as informações divulgadas pela Raízen, se conectam a 14 metas dos 17 ODS, conforme orienta a Agenda (Plataforma Agenda 2030, 2020).

Os Objetivos do Desenvolvimento Sustentável, que compõem a Agenda 2030, foram lançados em 2015, pela ONU (Nações Unidas, 2015), para acabar com a pobreza, proteger o meio ambiente e o planeta, bem como assegurar a prosperidade para todas as nações (ONU, 2015). Os ODS representam o núcleo da Agenda e deverão ser alcançados até o ano 2030.

Assim, na corrida contra o tempo, sabendo do desafio associado a esses compromissos, a Raízen lança edital para startups que auxiliem a atingir essas metas. Por meio do Pulse, o hub de inovação, exploram 13 iniciativas e algumas já estão em fase de testes nas operações, prometendo acelerar essa jornada, uma vez que, conforme a programação, deverão se esforçar para buscar o equilíbrio entre as três dimensões do desenvolvimento sustentável: a econômica, a social e ambiental.

\section{Considerações Finais}

A busca pela sustentabilidade estimula comportamentos, visões, novas práticas de gestão e entrevê novas oportunidades acrescentando valores aos produtos em harmonia com meio ambiente. Utilizando-se sempre da inovação e possibilitando a redução da procura de recursos naturais com a recuperação de desperdícios e resíduos, a Economia circular aviva perspectivas a serem encaradas pelas empresas para um crescimento com bases sólidas e com futuro promissor, além das vantagens competitivas no contexto de um mercado dinâmico.

De acordo com Weetman (2019), a tecnologia e as inovações digitais oferecem potencial extraordinário para promover a economia circular. A velocidade da mudança, porém, está acelerando, e as empresas precisam investir antes dos concorrentes: "Aprendemos isso no mundo da Internet do consumidor: quando é óbvio, é tarde demais. Isso significa que agora é a hora de agir. Você precisa perceber que estamos nos primeiros dois minutos de uma partida de futebol; a metade da partida já é tarde demais", afirma Bill Ruh, CEO da GE Digital, EUA.44. No entanto, esse é o grande diferencial da empresa estudada, são características priorizadas, que a coloca em posição de destaque no ramo.

A empresa em seus relatórios demonstra grande responsabilidade com o meio ambiente, com a sociedade e a economia, divulgando relevantes compromissos públicos. Apresenta estratégias como: a busca de visão integrada entre ambiental, social e de governança; trabalhando ativamente para gerar impactos socioambientais positivos; reconhecendo os impactos negativos e agindo continuamente para mitigá-los; com um potencial de produção e distribuição de energia limpa em escala global; tendo em vista sempre eficiência nos processos entregando resultados consistentes.

Sabe-se quão desafiador é gerir impactos positivos e negativos de toda grande empresa, mas observa-se nas divulgações, que não falta na Raízen, energia para pensar diferente, abraçar as oportunidades, quebrar as barreiras encontradas e ir além, gerando um esforço coletivo para a preservação dos nossos recursos naturais, para assim, ser referência global em bioenergia, protagonista na transição energética e destaque por buscar redefinir o futuro da energia.

\section{Referências}

Agencia Brasil (2021). https://agenciabrasil.ebc.com.br/economia/noticia/2021-05/empresas-apostam-em-economia-circular-para-reduzir-impactos-ambientais.

Brito, H. C., da silva, M. N., de brito, Y. M. A., portela, L. J. P., \& sasaki, J. K. (2021) Meio ambiente e sustentabilidade pesquisa, reflexões e diálogos emergentes. https://ampllaeditora.com.br/books/2021/06/eBook-Meio-Ambiente-e-Sustentabilidade-Vol-1.pdf.

Brundtland, G. H. (1987). Our Common Future -The World Commission on Environment and Development-Oxford University, Oxford University Press. 
Comissão Mundial Sobre Meio Ambiente E Desenvolvimento (CMMAD) (1988). Nosso futuro comum. Fundação Geteúlio Vargas.

Cosenza, J. P., Andrade, E. M., \& Assunção, G. M. (2020). Economia circular como alternativa para o crescimento sustentável brasileiro: análise da Política Nacional de Resíduos Sólidos. Rev. Gest. Ambient. e Sust. - GeAS, 9(1), 1-30, e16147. https://doi.org/10.5585/geas.v9i1.16147.

Ellen MacArthur Foundation. (2013) Towards the Circular Economy. Economic and business rationale for an accelerated transition. 1. $10.1162 / 108819806775545321$

Ellen MacArthur Foundation. (2017). Mission and Vision: Accelerating the transition to a circular economy. Ellen MacArthur Foundation - Our Story: https://www.ellenmacarthurfoundation.org/our-story/mission.

Freire, A. \& Fontgalland, I. (2021) Diversificação no setor de energia: o caso da Raízen, E-Acadêmica, 2(3), e282362. https://doi.org/10.52076/eacad-v2i3.62.

Fontgalland, I. Slides MEC, 2021, UFCG.

Gil, A. C. (2002). Como elaborar projetos de pesquisa (4a ed.). Atlas.

Lemos, P. (2018). Economia Circular como fator de resiliência e competitividade na região de Lisboa e Vale do Tejo: estudos para uma região RICA - resiliente, inteligente, circular e atractiva. Lisboa :Comissão de Coordenação e Desenvolvimento Regional de Lisboa e Vale do Tejo.

Nunes, M.G. (2020). Economia circular e as cadeias de abastecimento: estudo de caso. (Dissertação de Mestrado, Programa de Pós- graduação em gestão e estratégia industrial). Lisbon school of economics \& management. Universidade de Lisboa.

ONU - Organização das Nações Unidas. (2015). Objetivos de Desenvolvimento Sustentável da ONU. https://sustainabledevelopment.un.org/sdgs.

ONU - Organização das Nações Unidas. (2015). Transformando nosso mundo: a Agenda 2030 para o desenvolvimento sustentável. <https://goo.gl/jcFMVC〉.

Plataforma Agenda 2030. (2021). Os 17 Objetivos de Desenvolvimento Sustentável. http://www.agenda2030.org.br/os ods/.

Popadiuk, S. (2015) Estudos de casos - Metodologia baseada em Yin (2003 -2011).

Raízen (2021) -. https://www.raizen.com.br/sobre-a-raizen.

Ramos, C.; Zilber, M. A.; Freitas, L. F. S., \& Lhama, P. G. (2020) Economia circular como caminho para inovação e sustentabilidade. Revista Livre de Sustentabilidade e Empreendedorismo.

Richardson, R. J. (1999) Pesquisa social: métodos e técnicas. Atlas.

Silva, F. A da. (2019). Diagnostico de economia circular no complexo industrial de base florestal da região da lages/SC.116p. (Dissertação de Mestrado, Programa de Pós- graduação em Ciencias Ambientais) da Universidade do Estado de Santa Catarina.

Weetman, C. (2019) Economia Circular: conceitos e estratégias para fazer negócios de forma mais inteligente, sustentável e lucrativa. Tradução Afonso Celso da cunha serra. Autentica Business. 